schränkt. Werden die Erkenntnisse aus RCT durch breit angelegte Registerstudien bestätigt, kann ein Effekt als gesichert angesehen werden. Dies ist für die präventive Wirkung von Lithium bezüglich Suiziden und Suizidversuchen der Fall. Lithium hat damit lebensrettende Eigenschaften, wie sie weder andere zur Phasenprophylaxe eingesetzte Substanzen noch Antidepressiva [3] aufweisen. Die S3-Leitlinie Bipolare Störungen, die mit einer seltenen A-Empfehlung Lithium für Patienten mit hohem Suizidrisiko empfiehlt, erfährt durch die Studie von Hayes et al. weitere Unterstützung. Angesichts seiner überlegenen phasenprophylaktischen und seiner einzigartigen lebensrettenden Eigenschaften wird Lithium nach wie vor zu selten verordnet. Keinem bipolar affektiv erkrankten Patienten sollte ohne triftigen Grund die Chance einer Lithium-Therapie vorenthalten werden.
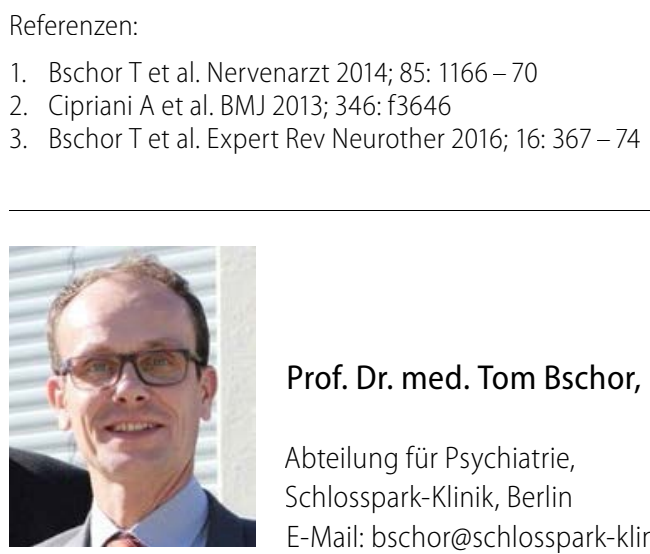

Prof. Dr. med. Tom Bschor, Berlin

Abteilung für Psychiatrie,

Schlosspark-Klinik, Berlin

E-Mail: bschor@schlosspark-klinik.de

\title{
Intratympanale Gabe von Methylprednisolon genauso wirksam wie von Gentamicin
}

Fragestellung: Ist die intratympanale Gabe von Methylprednisolon bei der Behandlung des Morbus Menière genauso wirksam wie die etablierte Therapie mit Gentamicin?

Hintergrund: Die Menièrsche Erkrankung geht mit heftigen Schwindelattacken und zunehmender Hörminderung einher. Ein klassischer Therapieansatz ist die intratympanale Gabe von Gentamicin. Dies führt dosisabhängig zu einem Untergang der vestibulären Rezeptoren und damit zu einem Ausfall der vestibulären Funktionen. Bei etwa $20 \%$ der so behandelten Patienten kommt es auch zu einer Hörminderung oder sogar zu einem Hörverlust. Daher ist es dringend notwendig, andere The-

Patel M, Agarwal K, Arshad Q et al. Intratympanic methylprednisolone versus gentamicin in patients with unilateral Ménière's disease: a randomised double-blind, comparative effectiveness trial. Lancet 2016; 388: $2753-62$ rapien zu entwickeln, bei denen die vestibulären Funktionen intakt bleiben und insbesondere kein Risiko für eine Hörminderung besteht.

Patienten und Methodik: Es handelt sich um eine doppel- blinde Therapiestudie bei Patienten im Alter zwischen 18 und 70 Jahren und therapierefraktärer unilateraler Menièrscher Erkrankung. Sie erhielten entweder zwei intratympanale Injektionen mit $62,5 \mathrm{mg} / \mathrm{ml}$ Methylprednisolon oder $40 \mathrm{mg} / \mathrm{ml}$ Gentamicin im Abstand von zwei Wochen. Die Beobachtungszeit betrug zwei Jahre. Der primäre Studienendpunkt war die Häufigkeit von Schwindelattacken über die letzten sechs Monate der Beobachtungszeit gegenüber den sechs Monaten vor der ersten Therapie.

Ergebnisse: Insgesamt wurden 60 Patienten randomisiert. In der Gentamicin-Gruppe kam es zu einem Rückgang der Schwindelattacken von 19,9 in den sechs Monaten vor Therapie auf 2,5 in den letzten sechs Monaten der Beobachtungszeit. Dies entspricht einer Reduktion von $87 \%$. In der MethylprednisolonGruppe kam es zu einem Rückgang der Schwindelattacken von 16,4 auf 1,6, entsprechend einer Reduktion um $90 \%$. Die Behandlung wurde sehr gut vertragen.

Schlussfolgerungen: Die intratympanale Gabe von Methylprednisolon ist bei der Menièrschen Erkrankung ebenso wirksam wie die Gabe von Gentamicin.

\section{- Kommentar von Hans-Christoph Diener, Essen}

\section{Die Patienten auf diese Therapieoption aufmerksam machen}

Diese Therapiestudie ist sehr wichtig, denn sie zeigt, dass auch die intratympanale Gabe von Methylprednisolon in der Behandlung der Menièrschen Erkrankung wirksam ist. In dieser Studie gab es bezüglich Hörstörungen zwischen den beiden Therapiegruppen keine Unterschiede. Die vestibulären Funktionstests zeigten allerdings eine erhaltene vestibuläre
Funktion bei den Patienten, die mit Methylprednisolon behandelt wurden, im Vergleich zu denjenigen, die mit Gentamicin behandelt wurden. Diese Erkenntnis kann direkt in die Praxis umgesetzt werden. Patienten mit Menièrscher Erkrankung sollten ab sofort auf diese neue Therapiemöglichkeit aufmerksam gemacht werden. 